\title{
Simulation of relativistic shocks and associated radiation from turbulent magnetic fields
}

\author{
K.-I. Nishikawa ${ }^{1}$, J. Niemiec ${ }^{2}$, M. Medvedev ${ }^{3}$, B. Zhang ${ }^{4}$, P. Hardee ${ }^{5}$,

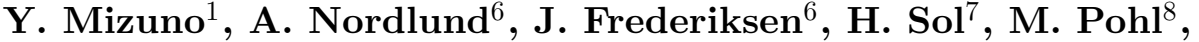 \\ D. H. Hartmann ${ }^{9}$ and G. J. Fishman ${ }^{10}$ \\ ${ }^{1}$ Center for Space Plasma and Aeronomic Research, University of Alabama in Huntsville, \\ NSSTC, 320 Sparkman Drive, Huntsville, AL 35805, USA \\ email: ken-ichi.nishikawa-1@nasa.gov \\ ${ }^{2}$ Institute of Nuclear Physics PAN, ul. Radzikowskiego 152, 31-342 Krakow, Poland \\ ${ }^{3}$ Department of Physics and Astronomy, University of Kansas, KS 66045, USA \\ ${ }^{4}$ Department of Physics and Astronomy, University of Nevada, Las Vegas, NV 89154, USA \\ ${ }^{5}$ Department of Physics and Astronomy, The University of Alabama, Tuscaloosa, AL 35487, \\ USA \\ ${ }^{6}$ Niels Bohr Institute, Juliane Maries Vej 30, 2100 Kbenhavn, Denmark \\ ${ }^{7}$ LUTH, Observatore de Paris-Meudon, 5 place Jules Jansen, 92195 Meudon Cedex, France \\ ${ }^{8}$ Institut fuer Physik und Astronomie, Universitaet Potsdam, 14476 Potsdam-Golm, Germany \\ ${ }^{9}$ Department of Physics and Astronomy, Clemson University, Clemson, SC 29634, USA \\ ${ }^{10}$ NASA/MSFC, 320 Sparkman Drive, Huntsville, AL 35805, USA
}

\begin{abstract}
Recent PIC simulations of relativistic electron-positron (electron-ion) jets injected into a stationary medium show that particle acceleration occurs in the shocked regions. Simulations show that the Weibel instability is responsible for generating and amplifying highly nonuniform, small-scale magnetic fields and for particle acceleration. These magnetic fields contribute to the electron's transverse deflection behind the shock. The "jitter" radiation from deflected electrons in turbulent magnetic fields has different properties from synchrotron radiation calculated in a uniform magnetic field. This jitter radiation may be important for understanding the complex time evolution and/or spectral structure of gamma-ray bursts, relativistic jets in general, and supernova remnants. In order to calculate radiation from first principles and go beyond the standard synchrotron model, we have used PIC simulations. We will present detailed spectra for conditions relevant to various astrophysical sites of collisionless shock formation. In particular we will discuss application to GRBs and SNRs.
\end{abstract}

Keywords. instabilities, magnetic fields, shock waves, radiation mechanisms: general

\section{Relativistic PIC Simulations}

Particle-in-cell (PIC) simulations can shed light on the physical mechanism of particle acceleration that occurs in the complicated dynamics within relativistic shocks. Recent PIC simulations of relativistic electron-ion and electron-positron jets injected into an ambient plasma show that acceleration occurs within the downstream jet (e.g., Nishikawa et al. 2009b). In general, these simulations have confirmed that the Weibel instability, which generates current filaments and associated magnetic fields, mediates the relativistic collisionless shock (Medvedev 1999), and accelerates electrons (Hededal 2005; Nishikawa et al. 2003, 2005, 2006; Ramirez-Ruiz, Nishikawa, \& Hededal 2007; Chang, Spitkovsky, \& Arons 2008; Spitkovsky 2008a,b; Sironi \& Spitkovsky 2009a; Nishikawa et al. 2009a). Therefore, the investigation of radiation resulting from accelerated particles (mainly electrons and positrons) in turbulent magnetic fields is essential to understand the radiation 
and its observable spectral properties. In this report we present a numerical method for obtaining spectra from particles self-consistently traced in our PIC simulations that goes beyond the standard synchrotron model (Waxman 2006).

\section{Calculating Emission from Electrons Moving in Self-consistently Generated Magnetic Fields}

We calculate the radiation spectra directly from our simulations by integrating the expression for the retarded power, derived from Liénard-Wiechert potentials for a large number of representative particles in the PIC representation of the plasma (Jackson 1999; Hededal 2005; Nishikawa et al. 2009a,c; Sironi \& Spitkovsky 2009b; Frederiksen et al. 2010). In order to obtain the spectrum of the synchrotron/jitter emission, we consider an ensemble of electrons selected in the region where the Weibel instability has fully grown and where the electrons are accelerated in the self-consistently generated magnetic fields.

We have validated our numerical method by performing simulations using a small system with $\left(L_{\mathrm{x}}, L_{\mathrm{y}}, L_{\mathrm{z}}\right)=(645 \Delta, 131 \Delta, 131 \Delta)(\Delta=1$ : grid size $)$ and a total of $\sim 0.5$ billion particles (12 particles / cell/species for the ambient plasma) in the active grid zones (Nishikawa et al. 2006). We first performed simulations without calculating radiation up to $t=450 \omega_{\mathrm{pe}}^{-1}$ when the jet front is located at about $x=480 \Delta$. We randomly selected 16,200 jet electrons near the jet front and calculated the emission during the sampling time $t_{\mathrm{s}}=t_{2}-t_{1}=75 \omega_{\mathrm{pe}}^{-1}$ with Nyquist frequency $\omega_{\mathrm{N}}=1 / 2 \Delta t=200 \omega_{\mathrm{pe}}$ where $\Delta t=0.005 \omega_{\mathrm{pe}}^{-1}$ is the simulation time step and the frequency resolution $\Delta \omega=1 / t_{\mathrm{s}}=$ $0.0133 \omega_{\text {pe }}$.

The spectra shown in Figure 1 are obtained for head-on $\left(\theta=0^{\circ}\right)$ emission from the jet electrons. The radiation from shows a Bremsstrahlung-like spectrum for the three cases shown (Hededal 2005). Since the magnetic fields generated by the Weibel instability are rather weak and jet electron acceleration is modest, the electron trajectories bend only slightly. For the case with $\gamma=15$ and $B_{\mathrm{x}}=3.7$ (simulation units), the spectrum is similar to that of a case without magnetic field. However, for the cases with $\gamma=7.08$ the spectrum in the case with $B_{x}=3.7$ shows an enhanced amplitude at higher frequencies. At this smaller Lorentz factor the Weibel instability grows to larger amplitude (the growth time is proportional to $(\gamma)^{1 / 2}$ ) than for the $\gamma=15$ and $B_{\mathrm{x}}=3.7$ case. In this case the transverse magnetic fields generated by the Weibel instability combined with

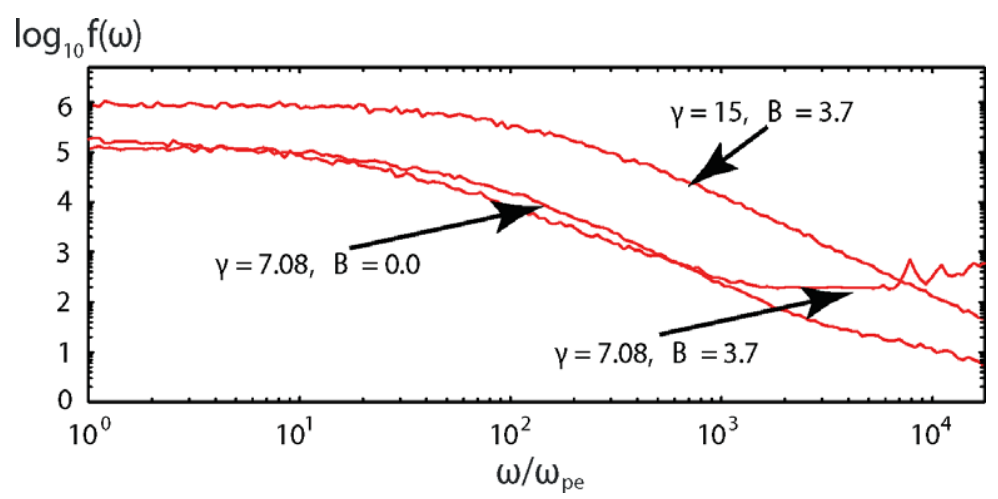

Figure 1. Spectra obtained from hot jet electrons $\left(v_{\mathrm{th}}^{\mathrm{jet}}=0.33 c\right)$ for three different cases $\left(\gamma=15, B_{x}=3.7, \gamma=7.08, B_{x}=3.7\right.$, and $\left.\gamma=7.08, B_{x}=0.0\right)$ for viewing angle $0^{\circ}$. 
the (longitudinal) ambient magnetic field bend the jet electron trajectories significantly to generate the higher frequencies.

Like a Bremsstrahlung spectrum, the lower frequencies have flat spectra and the higher frequencies decrease monotonically (Nishikawa et al. 2009C). However, the higher frequency slopes in Figure 1 are less steep than that in the Bremsstrahlung spectrum. This is due to the fact that the spread of Lorentz factors of jet electrons is larger and the average Lorentz factor is larger as well. Furthermore, even though the magnetic field strength is not so large, the spectra seem to be extended to higher frequency. This is explained that as shown in Fig. 7.16 (left) in Hededal?s Ph. D. thesis (Hededal, 2005) the turbulent magnetic field shifts the frequency higher with shorter wave length (smaller $\mu$ ). We obtained several different parameters with jet electrons and ambient magnetic field using a small system as in this report. However, the strength of the magnetic fields generated by the Weibel instability is small, therefore the spectra for these cases are very similar to the Bremsstrahlung spectrum.

Figure 2 shows how our synthetic spectrum matches with spectra obtained from Fermi observations. Figure 2a shows the model spectra for five time intervals (Abdo et al. 2009). The red line shows the slope $(a=1)$ in Fig. 2a and except the spectrum for the interval "a" the slopes for all other time intervals are approximately 1 . This is similar to a Bremsstrahlung-like spectrum at least for the low frequency side. As shown in Fig. $2 \mathrm{~b}$ the slope in the low frequency is very similar to the observed spectra. The peaks and slopes at high frequencies change over time.

\section{Discussion}

Emission obtained using the method described above is obtained self-consistently, and automatically accounts for magnetic field structures on the small scales responsible for jitter emission. By performing such calculations for simulations using different parameters, we can investigate and compare the different regimes of jitter- and synchrotron-type emission (Medvedev 2000, 2006). Thus, we should be able to address the low frequency GRB spectral index violation of the synchrotron spectrum line of death (Medvedev 2006).

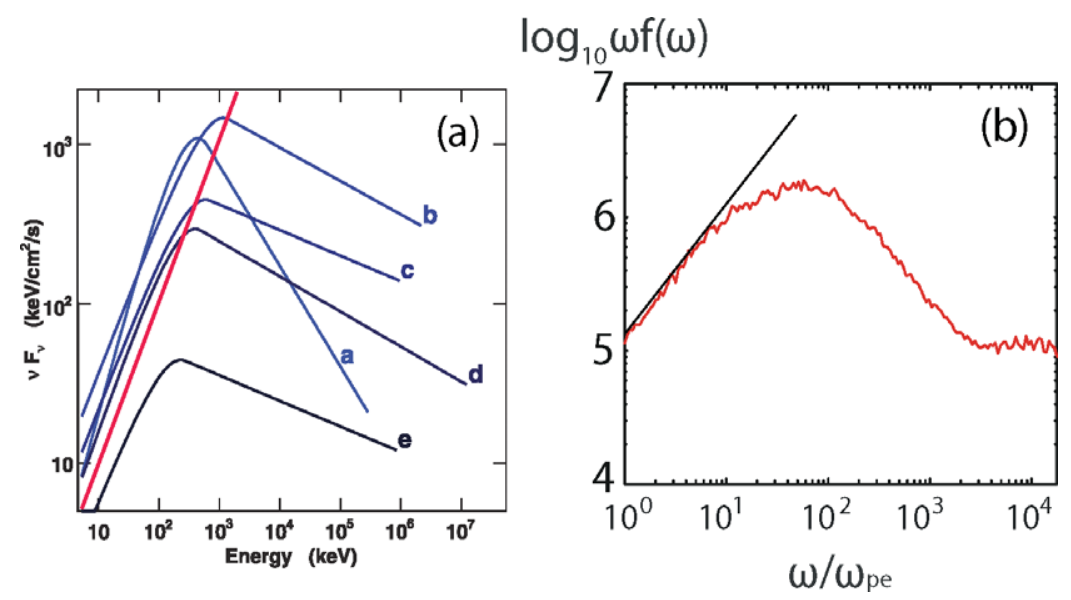

Figure 2. Brief comparison of a synthetic spectrum with spectra obtained from Fermi observations. Figure 2a shows the modeled Fermi spectra in $\nu F_{\nu}$ units for five time intervals. A flat spectrum would indicate equal energy per decade in photon energy. The changing shapes show the evolution of the spectrum over time. Figure $2 \mathrm{~b}$ shows the spectrum for the case of $\gamma=7.08$ with $B_{\mathrm{x}}=3.7$. The low frequency slope is approximately 1 and very similar to those spectra except interval "a". 
Recently, synthetic radiation has been calculated from simulations of laser-wakefield electron acceleration (Martins et al. 2009), counter-streaming jet (Frederiksen et al. 2010), and in shocks reflected at the wall (Sironi \& Spitkovsky 2009). In this study we inject relativistic jets into ambient plasma (from the left side of the computational box; see also Nishikawa et al. 2009b). A Weibel instability-mediated shock is formed and electrons become accelerated. We calculate the radiation from jet electrons in the observer frame, therefore calculated spectra can be directly compared with observations.

Behind the trailing shock the electrons are accelerated and strong magnetic fields are generated (Nishikawa et al. 2009b). Therefore, this region seems to produce the emission that is observed by satellites. We will examine the observed spectrum changes over time using different plasma conditions such as jet Lorentz factors, jet thermal temperatures, plasma compositions and other parameters.

\section{Achknowledgments}

This work is supported by NSF-AST-0506719, AST-0506666, AST-0908040, AST-0908010, NASA-NNG05GK73G, NNX07AJ88G, NNX08AG83G, NNX08AL39G, and NNX09AD 16G. JN was supported by MNiSW research project N N203 393034, and The Foundation for Polish Science through the HOMING program, which is supported through the EEA Financial Mechanism. Simulations were performed at the Columbia facility at the NASA Advanced Supercomputing (NAS). and IBM p690 (Copper) at the National Center for Supercomputing Applications (NCSA) which is supported by the NSF. Part of this work was done while K.-I. N. was visiting the Niels Bohr Institute. Support from the Danish Natural Science Research Council is gratefully acknowledged. This report was finalized during the program "Particle Acceleration in Astrophysical Plasmas" at the Kavli Institute for Theoretical Physics which is supported by the National Science Foundation under Grant No. PHY05-51164.

\section{References}

Abdo, A. A., et al. 2009, Science, 323, 1688

Chang, P., Spitkovsky, A., \& Arons, J. 2008, ApJ, 674, 378

Frederiksen, J. T., Haugboelle, T., Medvedev, M. V., \& Nordlund, Å. 2010, ApJ, 722, L114

Hededal, C. B., Ph.D. thesis, 2005, (arXiv:astro-ph/0506559)

Hededal, C. B. \& Nishikawa, K.-I. 2005, ApJ, 623, L89

Jackson, J. D., Classical Electrodynamics, Interscience, 1999.

Martins, J. L., Martins, S. F., Fonseca, R. A. Silva, L. O. 2009, Proc. of SPIE, 7359, 73590V-1

Medvedev, M. V. \& Loeb, A. 1999, ApJ, 526, 697

Medvedev, M. V. 2000. ApJ, 540, 704

Medvedev, M. V. 2006, ApJ, 637, 869

Nishikawa, K.-I., et al. 2003, ApJ, 595, 555

Nishikawa, K.-I., et al. 2005, ApJ, 623, 927

Nishikawa, K.-I., Hardee, P., Hededal, C. B., \& Fishman, G. J. 2006, ApJ, 642, 1267

Nishikawa, K. -I., et al. 2009a, AIPCP, 1085, 589

Nishikawa, K.-I., et al. 2009b, ApJ, 689, L10

Nishikawa, K.-I., et al. 2009c, AdvSR, submitted, (arXiv:0906.5018)

Ramirez-Ruiz, E., Nishikawa, K.-I., \& Hededal, C. B. 2007, ApJ, 671, 1877

Sironi, L. \& Spitkovsky, A. 2009a, ApJ, 698, 1523

Sironi, L. \& Spitkovsky, A. 2009b, ApJ, 707, L92

Spitkovsky, A. 2008a, ApJ, 673, L39

Spitkovsky, A. 2008b, ApJ, 682, L5

Waxman, E. 2006, Plasma Phys. Control. Fusion, 48, B137 (arXiv:astro-ph/0607353) 